Vol. 43 (1991) [101-113]

\title{
A FACTOR THEOREM FOR LOCALLY CONVEX DIFFERENTLABILITY SPACES
}

\author{
Roger Eyland and Bernice Sharp
}

\begin{abstract}
The main result of this paper is that a continuous convex function with domain in a locally convex space factors through a normed space. In a recent paper by Sharp, topological linear spaces are categorised according to the differentiability properties of their continuous convex functions; we show that under suitable conditions the classification is preserved by linear maps. A technique for deducing results for locally convex spaces from Banach space theory is an immediate consequence. Examples are given and Asplund $C(S)$ spaces are characterised.
\end{abstract}

\section{INTRODUCTION}

The classification of Banach spaces according to the differentiability properties of their continuous convex functions began with Asplund [1] in 1968; similar classification theory for topological linear spaces originates in Sharp $[13,14]$.

The mapping theorems of the first section extend those of Asplund; conditions are given for a mapping to preserve a space's classification. Preserving $G_{\delta}$ sets is unexpectedly difficult; a topological theorem of Čoban and Kenderov [3] gives a result for Fréchet spaces. We prove the factor theorem: if $f$ is a continuous convex function with a convex open domain in a locally convex space $X$, then there exists a continuous seminorm $p$ such that $f$ factors through $X_{p}=X /$ ker $p$, which is a normed space with the topology induced by $p$.

The mapping and factor theorems enable us to derive results for $X$ from the spaces $X_{p}$. Strongest conclusions can be drawn for "Q-complete" and "bound covering" spaces (these concepts, and those used in the next paragraph, are defined shortly); for $S$ completely regular, we show that $C(S)$ has both of these properties.

Major results are: the set of Fréchet differentiability points of a continuous convex function with domain in a bound covering space is a $G_{\delta}$ set; for a Q-complete space $X$, MDS and GDS coincide, and if in addition $X$ is bound covering, FMDS, FDS and ASP coincide; with some special conditions, $C(S)$ is a differentiability space if and only if for every compact subset $A$ of $S, C(A)$ is a differentiability space of the same type; hence $C(S)$ is ASP if and only if every compact subset of $S$ is dispersed.

Received 15th February, 1990.

Copyright Clearance Centre, Inc. Serial-fee code: 0004-9729/91 \$A2.00+0.00. 


\section{Preliminaries}

The term function is used for a real valued map. For a topological linear space $X$ and an open convex subset $D$ of $X$, a function $f$ on $D$ is said to be convex whenever, for all $x, y \in D$ and for all $t \in[0,1]$,

$$
f(t x+(1-t) y) \leqslant t f(x)+(1-t) f(y)
$$

The continuous dual of a topological linear space $X$ is denoted by $X^{*} ;$ in this paper all topological spaces are Hausdorff unless otherwise indicated.

Let $U$ be an open subset of $X$ and $\mathcal{M}$ be a bornology on $X$, that is, a class of bounded subsets containing all singletons. A function $f$ on $U$ is $\mathcal{M}$-differentiable at $x \in U$ whenever there exists $u \in X^{*}$ such that, for all $M \in \mathcal{M}$, for all $\varepsilon>0$, there exists $\delta>0$, such that for all $y \in M$, for all $t: 0<|t|<\delta$,

$$
\left|\frac{f(x+t y)-f(x)}{t}-u(y)\right|<\varepsilon .
$$

The function $u$ is uniquely determined by $f$ and $x$ and is denoted by $f^{\prime}(x)$.

If $\mathcal{M}$ is the class of all bounded subsets of $X$ then $f$ is Fréchet differentiable at $x$. This is the weakest of all possible choices for the derivative which coincide, when $X$ is a normed space, with the standard Fréchet derivative. If $\mathcal{M}$ is the class of all singleton subsets of $X$ then $f$ is Gateaux differentiable at $x$.

REMARK 0.1. A continuous convex function $f$ is Gateaux differentiable at $x \in D$ if and only if for all $y \in X$,

$$
\phi(y)=\lim _{t \rightarrow 0} \frac{f(x+t y)-f(x)}{t}
$$

exists, that is, $\phi$ is linear and continuous.

By gauge is meant a function $g$ on $X$ with the properties:

(1) for all $x \in X, g(x) \geqslant 0$;

(2) for all $x \in X$, for all $t \geqslant 0, g(t x)=t g(x)$; and

(3) for all $x, y \in X, g(x+y) \leqslant g(x)+g(y)$.

A generic set in $D$ contains a dense $G_{6}$ subset of $D$.

A topological linear space, $X$, will be classified, using the following abbreviations, according to the differentiability properties of a specified class of convex functions on $\boldsymbol{X}$.

(1) ASP (WASP): Asplund (Weak Asplund): every continuous convex function with domain a nonempty open convex subset is Fréchet (Gateaux) differentiable on a generic subset of its domain. 
(2) FDS (GDS): Fréchet (Gateaux) Differentiability Space: every continuous convex function with domain a nonempty open convex subset is Fréchet (Gateaux) differentiable on a dense subset of its domain.

(3) FMDS (MDS): Fréchet Minkowski Differentiability Space (Minkowski Differentiability Space): every continuous gauge on the space is Fréchet (Gateaux) differentiable on a dense subset.

(4) We will add "[gen]" to FMDS or MDS to indicate that the differentiability occurs on a generic set.

(5) We will add "[s]" to any of the above to indicate that the set of differentiability points is a $G_{6}$ set.

The classes are not necessarily distinct. For Banach spaces, GDS and MDS are equivalent $([11,6.6]) ;$ ASP and FDS are also equivalent because the set of points of Fréchet differentiability of a continuous convex function with domain in a Banach space is a $G_{\delta}$ set $([11,1.25])$.

A map $T: X \rightarrow Y$ is said to be bound covering whenever the following condition holds: for every bounded subset $B$ of $Y$ there exists a bounded subset $A$ of $X$ such that $T[A] \supset B$.

A linear bound covering map of a locally convex space onto a normed space is open; a linear open map of a normed space onto a normed space is bound covering. If $T$ is continuous, linear and onto, and $X$ and $Y$ are Banach spaces, then $T$ is bound covering, however this is not necessarily true even for Fréchet spaces $X$ and $Y$. (See [8, Ex. 22I].)

\section{MAPPING THEOREMS}

Suppose that $X$ and $Y$ are locally convex spaces, that $T: X \rightarrow Y$ is a linear, continuous, onto map and that $f$ is a continuous convex function with domain in $Y$. It follows from the definitions and 0.1 that $f$ is Gateaux differentiable at $T(x)$ if and only if $f \circ T$ is Gateaux differentiable at $x$ and that if $f$ is Fréchet differentiable at $T(x)$ then $f \circ T$ is Fréchet differentiable at $x$. If $T$ is also bound covering, then $f$ is Fréchet differentiable at $T(x)$ if and only if $f \circ T$ is Fréchet differentiable at $x$.

Theorem 1.1. Let $X$ and $Y$ be locally convex spaces, and $T$ a continuous, linear, onto map.

(1) If $X$ is GDS (MDS) then so is $Y$.

(2) Suppose also that $T$ is bound covering. If $X$ is FDS (FMDS) then so is $Y$.

Proof: Let $f$ be continuous and convex on a nonempty open convex subset $U$ of $Y$. Then $f \circ T$ is continuous and convex on $D=T^{-1}[U] ; D$ is nonempty, open and convex. 
If $X$ is GDS then the set $G$ of Gateaux differentiability points of $f \circ T$ is dense in $D$. Since $T$ is continuous, $T[G]$, which is the set of Gateaux differentiability points of $f$, is dense in $U$.

The FDS, MDS and FMDS proofs are similar.

The WASP and ASP cases are in general more difficult and stronger hypotheses are placed on $X$. In the proof of 1.3 the following theorem of Coban and Kenderov [3, Theorem 2.2] is used.

THEOREM 1.2. Let $F: X \rightarrow Y$ be a continuous and open single valued mapping from the regular topological space $X$ onto the regular space $Y$. Let there exist a completely metrisable dense subset $Z$ of $X$. Then a $G_{\delta}$ set $Q \subset Z$ exists such that the restriction of $F$ on $Q$ is a homeomorphism and $F[Q]$ is a dense and $G_{\delta}$ subset of $Y$.

Theorem 1.3. Let $X$ be a Fréchet space, $Y$ a regular linear space, and let $T: X \rightarrow Y$ be continuous, linear, open and onto.

(1) If $X$ is WASP (MDS[gen]) then so is $Y$.

(2) Suppose $T$ is also bound covering. If $X$ is ASP (FMDS[gen]) then so is $Y$.

Proof: We prove the ASP case, the others are similar. Suppose that $f, U$ and $D$ are as in 1.1. If $X$ is ASP, then there exists a set of Fréchet differentiability points $G$ of $f \circ T$ which is dense and $G_{\delta}$ in $D$. It follows that $G$ is metrisable with a complete metric (Mazurkiewicz's Theorem [4, XIV, 8.3]). From 1.2, there exists a subset $G^{\prime}$ of $G$ such that $T\left[G^{\prime}\right]$ is dense and $G_{\delta}$ in $U$. Since $T$ is bound covering, $T\left[G^{\prime}\right]$ is contained in the set of Fréchet differentiability points of $f$ in $U$.

If $Y$ is a Fréchet space then any continuous, linear, onto map $T$ is open ([15, Theorem 1 of 11.2]).

\section{FACTOR THEOREM}

If $X$ is a locally convex space, its topology may be defined by a family $P$ of seminorms; this topology is the weakest that makes each seminorm continuous. The $p$-balls, $\left\{B_{p}(\varepsilon): p \in P, \varepsilon>0\right\}$, where

$$
B_{p}(\varepsilon)=\{x \in X: p(x)<\varepsilon\}
$$

form a subbase of neighbourhoods at the origin. For a fixed $p$, these balls give a seminorm topology on $X: p$-open and $p$-continuous mean open and continuous with respect to this (not necessarily Hausdorff) topology. 
If $p$ is a continuous seminorm on a locally convex space $X$, denote by $X_{p}$ the space $X /$ ker $p$ with the topology given by the norm induced by $p$. The canonical map $\pi_{p}: X \rightarrow X_{p}$ is continuous, linear and onto; $X_{p}$ has the quotient topology if and only if $\pi_{p}$ is open. If $X$ and $X_{p}$ are complete, $\pi_{p}$ is open ([15, 11.2 Lemma 2]); if $X$ is fully complete $X_{p}$ is a Banach space if and only if $\pi_{p}$ is open. Fully complete [12, VI, 2] is also known as "Ptak" or "B-complete"; Fréchet spaces are fully complete.

If $p$ and $q$ are continuous seminorms on a locally convex space $X$, define $p \prec q$ and $q \succ p$ to mean, there exists $k>0$ such that, for all $x \in X$,

$$
p(x) \leqslant k q(x)
$$

and define $p \asymp q$ to mean that

$$
p \prec q \text { and } q \prec p \text {. }
$$

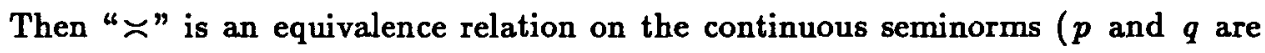
said to be equivalent seminorms if $p \asymp q$ ); "ঝ" is a partial order on the equivalence classes. Since $p \asymp q$ if and only if there exist $k, k^{\prime}>0$ such that, for all $x \in X$,

$$
k p(x) \leqslant q(x) \leqslant k^{\prime} p(x),
$$

in this case, $\operatorname{ker} p=\operatorname{ker} q ; X_{p}=X_{q}$ and $\pi_{p}=\pi_{q}$. Also

$$
k^{\prime} B_{p}(\varepsilon) \subset B_{q}(\varepsilon) \subset k B_{p}(\varepsilon),
$$

so $p$ and $q$ can be used interchangeably in a family of seminorms giving the topology on $X$. The meanings of $p$-open and $q$-open then coincide as do $p$-continuous and $q$-continuous.

The topology of a locally convex space may be defined by a family of $\asymp$ equivalence classes: take one or more representatives of each class and take the weakest topology that makes each of these seminorms continuous; the topology is independent of the choice of representatives and the quotients $X_{p}$, and maps $\pi_{p}$, depend only on the equivalence class. Also, $k p$ is a continuous seminorm whenever $p$ is, and $\max \{p, q\}$ defined by

$$
\max \{p, q\}(x)=\max \{p(x), q(x)\}
$$

is a continuous seminorm whenever $p$ and $q$ are, so we can always assume that a family of $\asymp$ equivalence classes of seminorms which generate the topology on $X$ is directed by $\prec$.

In this paper, defining family will mean a family of $\asymp$ equivalence classes of seminorms which form a directed set under the $\prec$ partial order: if $P$ is a defining family, we shall abuse notation and write $p \in P$ to mean that $p$ is a seminorm representing an equivalence class of $P$. 
LEMMA 2.1.

(1) Suppose that $X$ is a locally convex space, $D$ a convex open subset of $X$ and $f$ a continuous convex function on $D$. If, for a convex open balanced neighbourhood $U$ of 0 , and $M>0$,

$$
f[x+U]-f(x) \subset(-M, M)
$$

then, for all $\alpha \in[0,1]$,

$$
f[x+\alpha U]-f(x) \subset \alpha(-M, M) .
$$

(2) Suppose that $X$ is a linear space, $W$ a linear subspace of $X, f$ is convex on a nonempty convex subset $U$ of $X, x \in X$, and $x+W \subset U$. If $f$ is bounded above on $x+W$ then $f$ is constant on $x+W$.

Proof: For (1), by convexity of $f$, for all $\alpha \in[0,1]$,

$$
-\alpha(f(x-u)-f(x)) \leqslant f(x+\alpha u)-f(x) \leqslant \alpha(f(x+u)-f(x))
$$

and the result follows from $(*)$.

For (2), there exists $m \in \mathbf{R}$ such that for all $w \in W$,

$$
f(x+w) \leqslant m
$$

Let $r>1$, replace $\alpha$ by $\frac{1}{r}$ in $(* *)$ and let $u=r w$ : for all $w \in W$,

$$
-(m-f(x)) \leqslant r(f(x+w)-f(x)) \leqslant(m-f(x)) .
$$

If $f(x+w)-f(x) \neq 0$, taking $r$ sufficiently large provides a contradiction.

FACTOR THEOREM 2.2. Let $X$ be a locally convex space with a defining family $P$. Let $D$ be a nonempty open convex subset of $X$, and let $f$ be a convex function on $D$ continuous at $x_{0} \in D$. Then there exists $q \in P$ such that for all $p \succ q$,

(1) $D$ is $p$-open and

(2) $f$ is $p$-continuous on $D$.

Further, for each $p, D_{p}=\pi_{p}[D]$ is an open convex subset of $X_{p}$ and there exists a continuous convex function $f_{p}$ on $D_{p}$ such that

$$
f=f_{p} \circ \pi_{p}
$$

Proof: Since $D$ is open and $x_{0} \in D$, there is a $p_{1} \in P$ and an $\varepsilon_{1}>0$ such that $B_{p_{1}}\left(x_{0}, \varepsilon_{1}\right) \subset D$, (where $B_{p_{1}}\left(x_{0}, \varepsilon_{1}\right)$ denotes $x_{0}+B_{p_{1}}\left(\varepsilon_{1}\right)$ ). 
Since $f$ is continuous at $x_{0}$, there is a $p_{2} \in P$ and an $\varepsilon_{2}>0$ such that $f\left[B_{p_{2}}\left(x_{0}, \varepsilon_{2}\right)\right] \subset f\left(x_{0}\right)+(-1,1)$.

Let $q \succ p_{1}$ and $q \succ p_{2}$. Then for $p \succ q$ there is an $\varepsilon>0$ such that

$$
B_{p}\left(x_{0}, \varepsilon\right) \subset D
$$

and

$$
f\left[B_{p}\left(x_{0}, \varepsilon\right)\right] \subset f\left(x_{0}\right)+(-1,1) .
$$

Let $x \in D$. Since $D$ is open, there is a $t>0$ such that

that is

$$
\begin{aligned}
& z=(1+t) x-t x_{0} \in D \\
& x=\frac{1}{1+t} z+\frac{t}{1+t} x_{0}
\end{aligned}
$$

Let $u \in B_{p}(x, t /(1+t) \varepsilon)$. Then $u=x+t /(1+t) w$ for some $w \in B_{p}(0, \varepsilon)$, so that $x_{0}+w \in B_{p}\left(x_{0}, \varepsilon\right) \subset D$. So

$$
u=\frac{1}{1+t} z+\frac{t}{1+t}\left(x_{0}+w\right)
$$

is a convex linear combination of points of $D$ and so, since $D$ is convex, $u \in D$.

Thus for each $x \in D$ there is a $t>0$ such that $B_{p}(x, t /(1+t) \varepsilon) \subset D$, so $D$ is p-open.

Since $f$ is convex on $D$, if $u \in B_{p}(x, t /(1+t) \varepsilon)$

$$
\begin{aligned}
f(u) & =f\left(\frac{1}{1+t} z+\frac{t}{1+t}\left(x_{0}+w\right)\right) \\
& \leqslant \frac{1}{1+t} f(z)+\frac{t}{1+t} f\left(x_{0}+w\right) \\
& \leqslant \frac{1}{1+t} f(z)+\frac{t}{1+t}\left(f\left(x_{0}\right)+1\right)
\end{aligned}
$$

(from (*)). It follows that $f$ is bounded above on $B_{p}(x, t /(1+t) \varepsilon)$ and since $f$ is convex, there exists $M>0$ such that

$$
f\left[x+B_{p}\left(0, \frac{t}{1+t} \varepsilon\right)\right] \subset f(x)+(-M, M) .
$$

From Lemma $2.1(1)$ for each $\alpha \in(0,1]$

$$
f\left[x+\alpha B_{p}\left(0, \frac{t}{1+t} \varepsilon\right)\right] \subset f(x)+\alpha(-M, M) .
$$


To prove that $f$ is $p$-continuous on $D$ it suffices to show that for any $r>0$, there exists $\delta>0$, such that

$$
f\left[x+B_{p}(0, \delta)\right] \subset f(x)+(-r, r) .
$$

This is easily obtained from $(* *):$ let $r^{\prime}=\min \{r, M\}, \alpha=\frac{r^{\prime}}{M}$ and $\delta=\frac{r^{\prime}}{M} \frac{t}{1+t} \varepsilon$.

Since ker $p \subset B_{p}(0, \varepsilon), f[x+\operatorname{ker} p]$ is bounded which implies, from Lemma 2.1 (2), that $f$ is constant on $x+\operatorname{ker} p$, so $f_{p}: D_{p} \rightarrow \mathrm{R}$ is well defined by

$$
f_{p}(x+\operatorname{ker} p)=f(x)
$$

and $f=f_{p} \circ \pi_{p}$.

The function $f_{p}$ is continuous, because $f$ is $p$-continuous, and convex. $D_{p}$ is open, because $D$ is $p$-open, and is clearly convex.

REMARK. It is easy to see that Theorem 2.2 holds for gauges, that is, if $f$ is a gauge on $X$ then so is each $f_{p}$ on $X_{p}$.

Definitions. A locally convex space is said to be bound covering if there exists a defining family $P$ such that for all $p \in P, \pi_{p}$ is bound covering, and $Q$-complete (for "quotient complete") if there exists a defining family $P$ such that for all $p \in P, X_{p}$ is complete.

If $X$ is bound covering or Q-complete, we will assume that the defining family we use has the appropriate property. A large class of spaces with these properties is given in 3.6.

A complete bound covering space is Q-complete: if $X$ is bound covering each $\pi_{p}: X \rightarrow X_{p}$ is bound covering and open, since $X_{p}$ is normed; this means that $X_{p}$ has the quotient topology and so, since $X$ is complete, is a Banach space.

Theorems 1.1 and 1.3 can be used to transfer differentiability space properties from $X$ to each $X_{p}$.

Theorem 2.3. Let $X$ be a locally convex space and $P$ a defining family for $X$. Again, for $p \in P, \pi_{p}$ denotes the canonical map from $X$ to $X_{p}$.

(1) If $X$ is GDS (MDS) then for all $p \in P$, so is $X_{p}$.

(2) Suppose for some $p \in P, \pi_{p}$ is bound covering. If $X$ is FDS (FMDS), then so is $X_{p}$.

(3) If $X$ is a $Q$-complete Fréchet space which is WASP (MDS[gen]) then so is every $X_{p}$.

(4) If $X$ is a bound covering Fréchet space which is ASP (FMDS[gen]) then so is every $X_{p}$.

In 2.3 (3) the Q-completeness ensures that each $\pi_{p}$ is open. 


\section{The INHERITANCE OF NORMED SPACE PROPERTIES}

The following lemma highlights some facts which are used in the proof Theorems 3.2 and 3.3.

Lemma 3.1. Let $X$ be a locally convex space with a defining family $P$; let $f$ be a continuous convex function on $D$, a nonempty open convex subset of $X$; let $G$ $(F)$ be the set of Gateaux (Fréchet) differentiability points of $f$. For each seminorm $p \in P$, let $D_{p}=\pi_{p}[D]$. Let $q \in P$ be such that for all $p \succ q, f=f_{p} \circ \pi_{p}$ where $f_{p}$ is a continuous convex function on $D_{p}$; let $G_{p}\left(F_{p}\right)$ be the set of Gateaux (Fréchet) differentiability points of $f_{p}$.

(a) For all $p \succ q, G=\pi_{p}^{-1}\left[G_{p}\right]$.

(b) For all $p \succ q, F \supset \pi_{p}^{-1}\left[F_{p}\right]$.

(c) If $X$ is bound covering, then for all $p \succ q, F=\pi_{p}^{-1}\left[F_{p}\right]$.

(d) If $A$ is a $G_{\delta}$ subset of $D_{p}$, then $\pi_{p}^{-1}[A]$ is a $G_{\delta}$ subset of $D$.

(e) Let $\pi_{p}$ be open. (If $X$ is bound covering, or complete and Q-complete this condition is satisfied.) If $A_{p}$ is a dense set in $D_{p}$ then $\pi_{p}^{-1}\left[A_{p}\right]$ is dense in $D$.

(f) Let $A \subset D$ and suppose that for all $p \succ q$, there is a dense subset $A_{p}$ of $D_{p}$ such that $A \supset \pi_{p}^{-1}\left[A_{p}\right]$. Then $A$ is dense in $D$.

Proof: Parts (a), (b), and (c) are simple consequences of the remarks at the beginning of Section 1 ; (d) and (e) are immediate from the definitions. It only remains to establish ( $f$ ).

Let $x \in D$ and let $N$ be a neighbourhood of $x$. Then there is a $p \in P$ such that $x+B_{p}(\varepsilon) \subset N$; we may suppose, without loss of generality, that $p \succ q$ and $x+B_{p}(\varepsilon) \subset D$. Since $A_{p}$ is dense in $D_{p},\left(\pi_{p}(x)+B(\varepsilon)\right) \cap A_{p}$ is nonempty (where $B(\varepsilon)$ denotes the norm ball of radius $\varepsilon$ of $p$ in $\left.X_{p}\right)$. But

$$
\begin{aligned}
N \cap A & \supset\left(x+B_{p}(\varepsilon)\right) \cap A \\
& \supset \pi_{p}^{-1}\left[\pi_{p}(x)+B(\varepsilon)\right] \cap \pi_{p}^{-1}\left[A_{p}\right] \\
& =\pi_{p}^{-1}\left[\left(\pi_{p}(x)+B(\varepsilon)\right) \cap A_{p}\right] ;
\end{aligned}
$$

so $N \cap A$ is nonempty and $A$ is dense in $D$.

Since Banach spaces are clearly bound covering, Theorem 3.2 is a generalisation of the well known result that the set of Fréchet differentiability points of a continuous convex function with domain in a Banach space is a $G_{6}$ set.

THEOREM 3.2. If $f$ is a continuous convex function on an open convex subset $D$ of a bound covering space, the set of points of Fréchet differentiability $F$ of $f$ is a $G_{6}$ set in $D$. 
Proof: From Theorem 2.2, there exists $q \in P$ such that $f=f_{q} \circ \pi_{q}$ where $f_{q}$ is a continuous convex function on $D_{q}=\pi_{q}[D]$. Since $X_{q}$ is a normed space, the set $F_{q}$ of points of Fréchet differentiability of $f_{q}$ is a $G_{\delta}$ subset of $D_{q}([5$, p.155]), and from 3.1(c) and (d), $F=\pi_{q}^{-1}\left[F_{q}\right]$ is a $G_{\delta}$ subset of $D$.

In the statements of (2) and (3) of Theorem 3.3 below, "differentiability space" may be replaced by any of the previously defined classes.

THEOREM 3.3.

(1) If $X$ is a locally convex space which has a defining family $P$ such that for all $p \in P, X_{p}$ is GDS, MDS, FDS, FMDS, WASP[s] or MDS[s], then so is $X$.

(2) If $X$ is bound covering, with defining family $P$ such that for all $p \in P$, $X_{p}$ is a differentiability space, then $X$ is a differentiability space of the same type.

(3) If $X$ is complete and $Q$-complete, with defining family $P$ such that for all $p \in P, X_{p}$ is a differentiability space other than ASP[s] or FMDS[s], then $X$ is a differentiability space of the same type.

Proof: Let $f$ be a continuous convex function on $D$, a nonempty open convex subset of $X$. By Theorem 2.2 there is a $q \in P$ such that for all $p \succ q, f=f_{p} \circ \pi_{p}$ where $f_{p}$ is a continuous convex function on $D_{p}=\pi_{p}[D]$. The theorem then follows from the parts of Lemma 3.1 indicated.

In (1), (2) and (3), GDS and MDS follow from (a) and (f); FDS and FMDS from (b) and (f); WASP[s] and MDS[s] from (a), (d) and (f).

For (2) and (3), ASP and FMDS[gen] follow from (b), (d) and (e); WASP and MDS[gen] from (a), (d) and (e).

For (2), ASP[s] and FMDS[s] follow from (c), (d) and (e).

It is interesting to note that in Theorem 3.3, WASP[s] needs weaker hypotheses than WASP.

Theorem 3.3 permits us to use theorems which are well known for Banach or normed spaces to deduce results for locally convex spaces. In Theorem 3.4, we show that it suffices to test all continuous gauges; "Q-complete" is in fact a superfluous hypothesis but a proof is outside the scope of this paper, since results here are derived from Banach space theory.

TheOREM 3.4. For a $Q$-complete space $X, M D S$ and GDS are equivalent; if $X$ is also bound covering, FMDS, FDS and ASP are equivalent.

Proof: If $X$ is FMDS, then for all $p$, so is $X_{p}(2.3(2))$. Since each $X_{p}$ is a Banach space, each $X_{p}$ is FDS [5, p.158]. From 3.3 (1) and 3.2, $X$ is ASP. 
The MDS proof is similar, using the equivalence of MDS and GDS for Banach spaces $[11,6.6]$.

THEOREM 3.5. If $X$ is a bound covering, $Q$-complete space, with a defining family $P$ such that for all $p \in P, p$ is Fréchet differentiable everywhere except on ker $p$, then $X$ is $A S P$.

PRoof: This follows from 3.3 (2) and the fact that if the norm on a Banach space is Fréchet differentiable on $X \backslash\{0\}$ then the space is ASP [5, p.170].

The following example shows that a large class of spaces is both Q-complete and bound covering.

EXAMPLE 3.6. For a completely regular space $S$, denote by $C(S)$ the space of continuous functions on $S$ with the topology of compact convergence, that is, the topology generated by the family $p_{A}$ of seminorms, where

$$
p_{A}(x)=\sup _{t \in A}|x(t)|
$$

and $A$ ranges over all compact subsets of $S$. There is no loss of generality in assuming $S$ to be completely regular: for any topological space $X$ there is a completely regular $S$ such that $C(X)$ is linearly isomorphic to $C(S)[6,3.9]$.

Using [7, p.142 Theorem 11] and the method of proof of the Tietze extension theorem (see for example [4, p.149]), one can show that: if $S$ is a completely regular topological space, $A$ a compact subset of $S$ and $f: A \rightarrow[-c, c]$ a continuous function, then there is a continuous $\widetilde{f}: S \rightarrow[-c, c]$ such that $\left.\tilde{f}\right|_{A}=f$.

This means that the map $\gamma: C(S) / \operatorname{ker} p_{A} \rightarrow C(A)$ defined by

$$
\gamma\left(x+\operatorname{ker} p_{A}\right)=\left.x\right|_{A}
$$

is a linear isomorphism, so that we can identify $C(S)_{p_{A}}$ with $C(A)$. Since $A$ is compact, $C(A)$ with the topology generated by $p_{A}$ (that is, the sup norm topology) is a Banach space: it follows that $C(S)$ is Q-complete.

Let $M=\{x \in C(S):(\forall t \in S)(|x(t)|<1)\} ; M$ is bounded in $X$. If $\mathrm{B}$ is the unit ball in $C(A)$, the version of the Tietze extension theorem given above implies that $\pi_{p_{A}}[M] \supset B$. It follows that $\pi_{p_{A}}$ is bound covering and $C(S)$ is a bound covering space.

ThEOREM 3.7. For $S$ completely regular, $C(S)$ is a differentiability space if for all compact subsets $A$ of $S, C(A)$ is a differentiability space of the same type. The reverse implication holds except for WASP[s], MDS[s], WASP and MDS[gen]; the last two are true with the additional hypothesis that $C(S)$ be a Fréchet space. 
ProOF: Since $C(S)$ is bound covering, the first part is immediate from 3.3 (2). The converse follows from 2.3, noting that by 3.2, FDS, ASP and ASP[s] coincide, as do FMDS, FMDS[gen] and FMDS[s].

Namioka and Phelps [10] have shown that for $S$ compact, $C(S)$ is ASP if and only if $S$ is dispersed. The following corollary generalises their result.

Corollary 3.8. For $S$ completely regular, $C(S)$ is ASP if and only if every compact subset of $S$ is dispersed.

\section{REFERENCES}

[1] E. Asplund, 'Fréchet Differentiability of Convex Functions', Acta Math 121 (1968), 31-47.

[2] J.M. Borwein, 'Continuity and Differentiability Properties of Convex Operators', Proc. London Math. Soc. 3 (1982), 420-444.

[3] M. Coban and P. Kenderov, 'Generic Gateaux Differentiability of Convex Functionals in $C(T)$ and the Topological Properties of $T$ ', in Mathematics and Education in Mathematics, 1986: Proceedings of the Fifteenth Spring Conference of the Union of Bulgarian Mathematicians, pp. 141-149, 1986.

[4] J. Dugundji, Topology (Allyn and Bacon, Boston, 1970).

[5] J.R. Giles, Convex Analysis with Application in Differentiation of Convex Functions: Research Notes in Mathematics 58 (Pitman, 1982).

[6] L. Gillman and M. Jerison, Rings of Continuous Functions (Van Nostrand, Princeton, 1960).

[7] J.L. Kelley, General Topology: Graduate Texts in Mathematics 27 (Springer-Verlag, Berlin, Heidelberg, New York, 1955).

[8] J.L. Kelley and I. Namioka, Linear Topological Spaces (Van Nostrand, Princeton, N.J., 1963).

[9] D.G. Larman and R. Phelps, 'Gateaux Differentiabilty of Convex Functions on Banach Spaces', J. London Math. Soc (2) 20 (1979), 115-127.

[10] Namioka and R. Phelps, 'Banach Spaces which are Asplund Spaces', Duke Math. J. 42 (1975), 735-750.

[11] R. Phelps, Convex Functions, Monotone Operators and Differentiability: Lecture Notes in Mathematics 1364 (Springer-Verlag, Berlin, Heidelberg, New York, 1989).

[12] A.P. Robertson and W. Robertson, Topological Vector Spaces (Cambridge University Press, 1966).

[13] B. Sharp, Ph.D. Thesis (University of Newcastle, Australia, 1989).

[14] B. Sharp, 'The Differentiability of Convex Functions on Topological Linear Spaces', Bull. Austral. Math. Soc. 42 (1990), 201-213.

[15] A. Wilansky, Functional Analysis (Blaisdell, 1964). 
Department of Pure Mathematics University of Sydney New South Wales 2006 Australia
Australian Catholic University 40 Edward Street

North Sydney NSW 2060

Australia 